\title{
CDISC Rheumatoid Arthritis Therapeutic Area User Guide Version 1.0
}

National Cancer Institute

\section{Source}

National Cancer Institute. CDISC Rheumatoid Arthritis Therapeutic Area User Guide

Version 1.0. NCI Thesaurus. Code C161450.

The 1.0 version of the CDISC Rheumatoid Arthritis therapeutic area user guide. 